\title{
Synthesis of carbon nanotubes by the laser ablation method: Effect of laser wavelength
}

\section{Justyna Chrzanowska', Jacek Hoffman', Artur Małolepszy², Marta Mazurkiewicz' ${ }^{2}$, Tomasz A. Kowalewski', Zygmunt Szymanski', and Leszek Stobinski ${ }^{*, 3,4}$}

\footnotetext{
${ }^{1}$ Institute of Fundamental Technological Research, Polish Academy of Sciences, Pawinskiego 5B, Warsaw 02-106, Poland

${ }^{2}$ Faculty of Materials Science and Engineering, Warsaw University of Technology, Woloska 141, Warsaw 02-507, Poland

${ }^{3}$ Institute of Physical Chemistry, Polish Academy of Sciences, Kasprzaka 44/52, Warsaw 01-224, Poland

${ }^{4}$ University Research Centre "Functional Materials", Warsaw University of Technology, Woloska 141, Warsaw 02-507, Poland
}

Received 8 October 2014, revised 8 February 2015, accepted 6 March 2015

Published online 31 March 2015

Keywords carbon nanotubes, laser radiation fluence, pulsed laser vaporization, Raman spectra

*Corresponding author: e-mail Istobinski@ichf.edu.pl, Phone: +48 22343 3431, Fax: +48 226325276

The effect of laser wavelength on single-wall carbon nanotubes synthesis yield and their properties was studied. A doublepulse Nd:YAG laser, working at a wavelength of 355 or $1064 \mathrm{~nm}$, was used for carbon nanotubes production. The synthesized carbon nanotubes (CNTs) were investigated using the SEM/STEM microscopy and Raman spectroscopy. The results show that the useful range of UV laser radiation fluence is narrower and the properties of synthesized CNTs depend much more on the laser fluence than in the case of infrared laser radiation.
1 Introduction Carbon atoms have the unique ability to congregate into miscellaneous nanostructures under the conditions of laser vaporization. The covalent bonds between constituent carbon atoms are outstandingly strong which results in high mechanical stability and thermal conductivity of the macromolecular structures. These properties result in broad application of carbon nanostructures in nanotechnology. The pulsed laser vaporization (PLV) of graphite is used to obtain a wide variety of carbon allotropes, such as diamond-like carbon films [1], fullerene carbon molecules [2], carbon nanotubes [3-10], carbon nanowalls [11], nanohorns [12], and graphene [13-16].

Pulsed lasers are especially suitable in generation of non-equilibrium conditions due to a short time scale of temperature changes. PLV offers extraordinarily high temperatures in which graphite and metallic catalyst particles are transformed into the carbon-metal vapor. During the interaction of the laser beam with a target, its surface is heated to a temperature exceeding the boiling point and sometimes exceeding the critical temperature. At the same moment, vaporization begins. The evaporated particles are further heated by the laser pulse to temperatures of several tens of kilokelvin and form a plasma plume. Next, the expansion of the plasma plume takes place. When the plasma cools during expansion along a steep temperature gradient, carbon and catalyst metal atoms condense into larger structures. The conditions which initially change within a nanosecond time scale are far from equilibrium and enable synthesis of various nanostructures, otherwise, energetically impossible under equilibrium growth conditions [17-19].

Carbon nanotubes (CNTs) are among the most promising materials in nanotechnology. They have unique electrical, mechanical (e.g., the $\mathrm{C}-\mathrm{C}$ covalent bond in CNTs is among the strongest found in nature), and thermal properties, potentially with wide applications in electronics, polymer chemistry, computers, aerospace, and other industries. The nanotubes are obtained in several structural forms: single-wall CNTs (SWCNTs), double-wall CNTs, and multi-wall CNTs. They are mainly produced by the chemical vapor deposition (CVD) [19], the laser vaporization, and electric arc discharge methods. In the laser vaporization method, both continuous wave lasers [20-23] and pulsed lasers [3-10] are successfully used.

Single-wall CNTs produced by the PLV method are of excellent structural integrity, long, and of high purity. 
Usually, 1064 or $532 \mathrm{~nm} \mathrm{Nd:YAG} \mathrm{lasers} \mathrm{are} \mathrm{applied} \mathrm{[3-8]}$ although $248 \mathrm{~nm} \mathrm{KrF}$ lasers were also used with success [9].

In this paper, the effect of laser wavelength on SWCNTs properties and production yield was studied. A double-pulse $\mathrm{Nd}$ :YAG laser which delivers two subsequent pulses of the same wavelength was used for CNTs synthesis. This creates a unique opportunity to study the effect of laser wavelength, on the synthesis and properties of produced carbon nanotubes. The presented synthesis laser system was used for the first time. Usually, two separate lasers working at two different laser wavelengths, 532 and $1064 \mathrm{~nm}$ [8] and a dichroic mirror were used. In this experiment, the laser operated at a wavelength of 355 or $1064 \mathrm{~nm}$. The synthesized CNTs were investigated by the SEM/STEM techniques and Raman spectroscopy.

2 Experimental The reactor for synthesis of CNTs, assembled at the Institute of Fundamental Technological Research is shown in Fig. 1. This is a typical laser-furnace system for horizontal operation. The ablation of the target was performed in argon background gas at a pressure of $6.6 \times 10^{4} \mathrm{~Pa}$ flowing slowly $(\sim 5 \mathrm{~mm} / \mathrm{s})$ in a quartz tube $25 \mathrm{~mm}$ in diameter inserted in an outer $50 \mathrm{~mm}$ tube. The graphite target consists of raw natural graphite decorated with $\mathrm{Ni}$ and $\mathrm{Co}$ nanoparticles at a concentration of 1 at $\%$ for each of them. Metal particles were deposited by reducing of nickel and cobalt chlorides using $\mathrm{NaBH}_{4}$. The outer quartz tube is mounted inside a $42 \mathrm{~cm}$ long split-tube furnace operating at $1000{ }^{\circ} \mathrm{C}$. The target is situated in the center of the furnace. Evaporated by the laser pulse material was carried away by the argon stream and deposited on a watercooled brass collector located at the exit of the furnace. Graphite target irradiation is performed by a double-pulse Nd:YAG Ekspla 303 D laser. This is a two-laser system which provides two pulses of the same wavelength with adjustable inter-pulse delay time. The laser can be operated at a wavelength of 355,532 , and $1064 \mathrm{~nm}$ with $8 \mathrm{~ns}$ pulse duration with a repetition rate of $20 \mathrm{~Hz}$. After completion of the experiment, the carbon soot containing nanotubes was scraped off the collector surface and analyzed.
In this experiment, the laser was operated at a wavelength of 1064 or $355 \mathrm{~nm}$ in the fluence range of $1-$ $6 \mathrm{~J} \cdot \mathrm{cm}^{-2}$. The fluence of both pulses was usually similar, the second pulse being delayed by $30 \mathrm{~ns}$. Therefore, in the following part of this paper, fluence $F$ denotes fluence of each of two pulses; total fluence is two times larger. On the other hand, it is not reasonable to add fluence of both pulses, as was done in some analyses, because two pulses delayed by $30 \mathrm{~ns}$ cannot be regarded as just one longer pulse. The second pulse is absorbed by the earlier ablated plume, which decreases its interaction with the target. Gated ICCD images of the plume emission in ambient argon at a pressure of $7.3 \times 10^{4} \mathrm{~Pa}$ show that already at delay times of $30 \mathrm{~ns}$ following laser ablation of graphite, well-developed $250 \mu \mathrm{m}$ thick plume is formed [24]. This plasma plume strongly absorbs laser radiation and significantly changes energy transfer from laser pulse to the target. Consequently, the second pulse heats the plume more than the target.

In order to obtain a large spot on the target, the laser beam was defocused which resulted in a beam intensity distribution similar to a top-hat distribution. Two typical experimental configurations $[6,8]$ were used: configuration 1 with a single outer tube only and configuration 2 with a double inner and outer tubes.

\section{Results and analysis}

3.1 Laser wavelength of $1064 \mathrm{~nm}$ The results for configuration 1 and $1064 \mathrm{~nm}$ laser wavelength are shown in Figs. 2-4. Experimental conditions were as follows: the first pulse energy was $25 \mathrm{~mJ}$ (fluence $1 \mathrm{~J} \cdot \mathrm{cm}^{-2}$ ) while the second pulse energy varied from 16 to $32 \mathrm{~mJ}$ (fluence $0.65-1.3 \mathrm{~J} \cdot \mathrm{cm}^{-2}$ ). The variation of the second pulse energy in this range had no significant influence on the results.

Figure 2 shows SEM/STEM images of the collected soot with CNTs, while Fig. 3 presents images of bundled SWCNTs. The SEM/STEM images were collected using a Hitachi s5500 high resolution field emission microscope. They show the synthesized CNTs and impurities of residual catalysts incorporated in the carbon material. The diameter of the obtained CNTs presented in the STEM images is about $1.25 \mathrm{~nm}$, which can be estimated as the distance

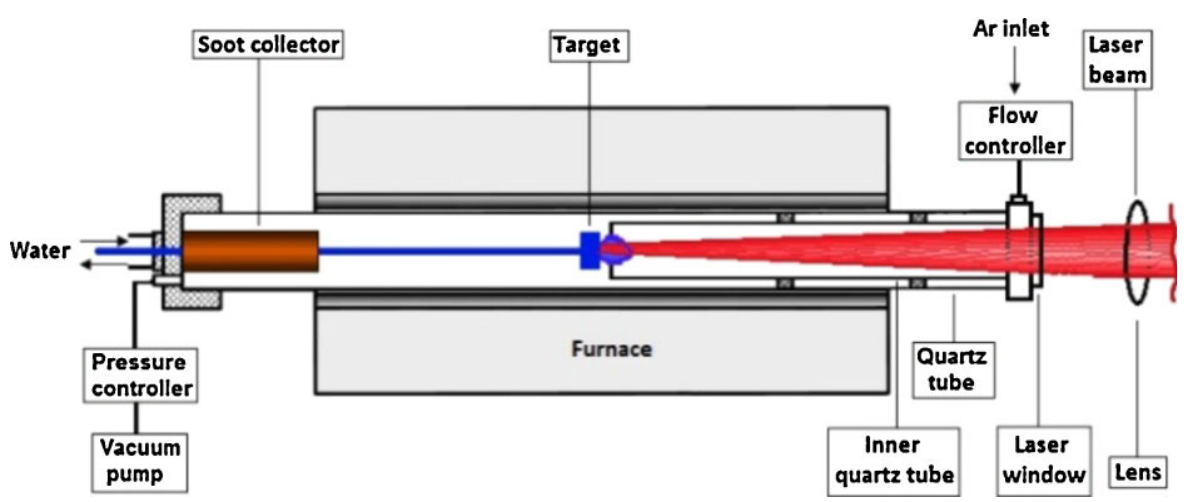

Figure 1 Reactor for carbon nanotube synthesis by the laser ablation method. 


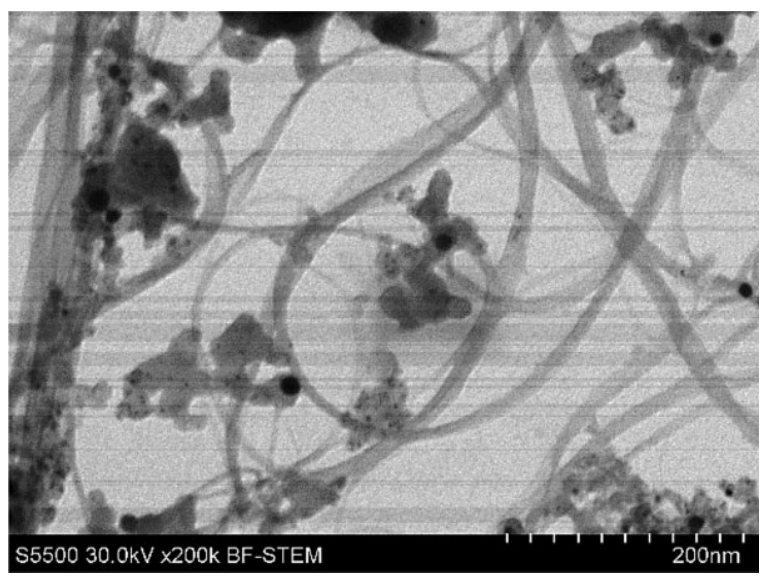

Figure 2 STEM image of carbon soot containing carbon nanotubes.

between two dark lines visible in a STEM image (Fig. 3) of SWCNT bundles.

The Raman spectra are shown in Fig. 4. These measurements were carried out using a laser excitation wavelength of $514.5 \mathrm{~nm}$ corresponding to the excitation energy of $2.41 \mathrm{eV}$.
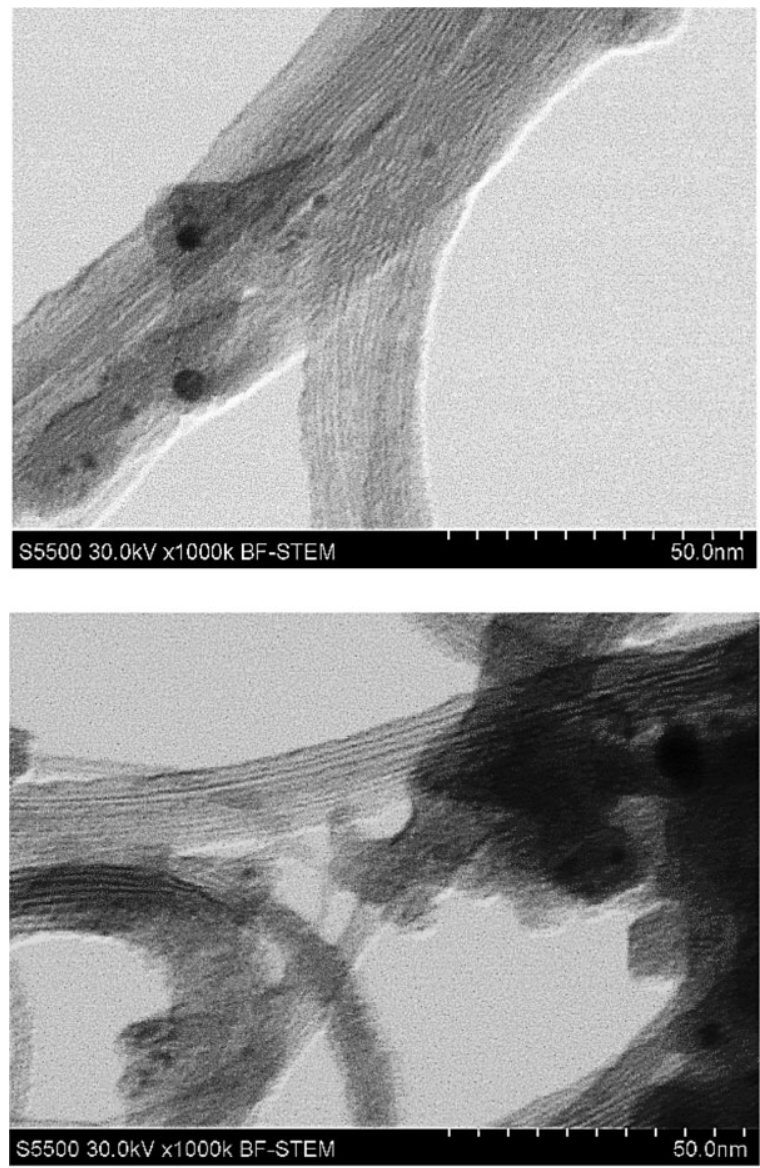

Figure 3 Images of bundled SWCNTs. Black dots show the metal nanoparticles (catalyst).
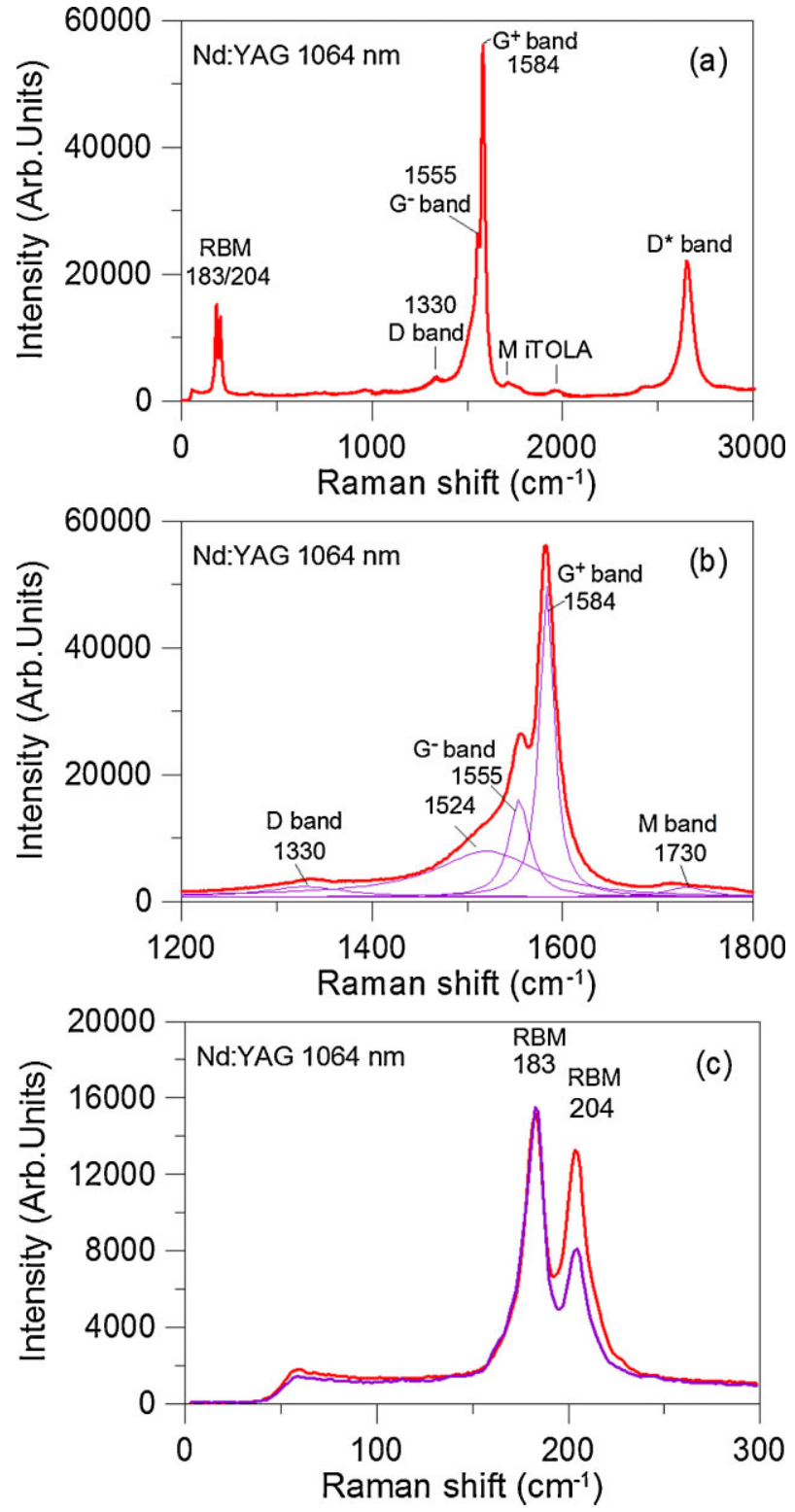

Figure 4 Raman shifts for PLV (configuration 1) with Nd:YAG $1064 \mathrm{~nm}$ at $F=1 \mathrm{~J} \cdot \mathrm{cm}^{-2}$; (a) full spectrum, (b) deconvoluted $\mathrm{G}$ bands, (c) RBMs.

The total spectrum (Fig. 4a) shows the radial breathing mode (RBM) band, which corresponds to the radial expansion-contraction of nanotube, the $\mathrm{D}$ (disorder band) and its second-order overtone the $\mathrm{D}^{*}$ band (sometimes denoted as $\mathrm{G}^{\prime}$ ), graphitic $\mathrm{G}^{-}$and $\mathrm{G}^{+}$bands as well as weak double resonance features associated with the $\mathrm{M}$ band and the iTOLA second-order modes [25].

Figure $4 \mathrm{~b}$ shows the position and shape of the graphitic peaks $\mathrm{G}^{-}$and $\mathrm{G}^{+}$after deconvolution. The lower frequency component $\mathrm{G}^{-}$is highly relevant for analysis because its profile and position strongly depends on the nature of SWCNTs. In the case of semi-conducting nanotubes, its 

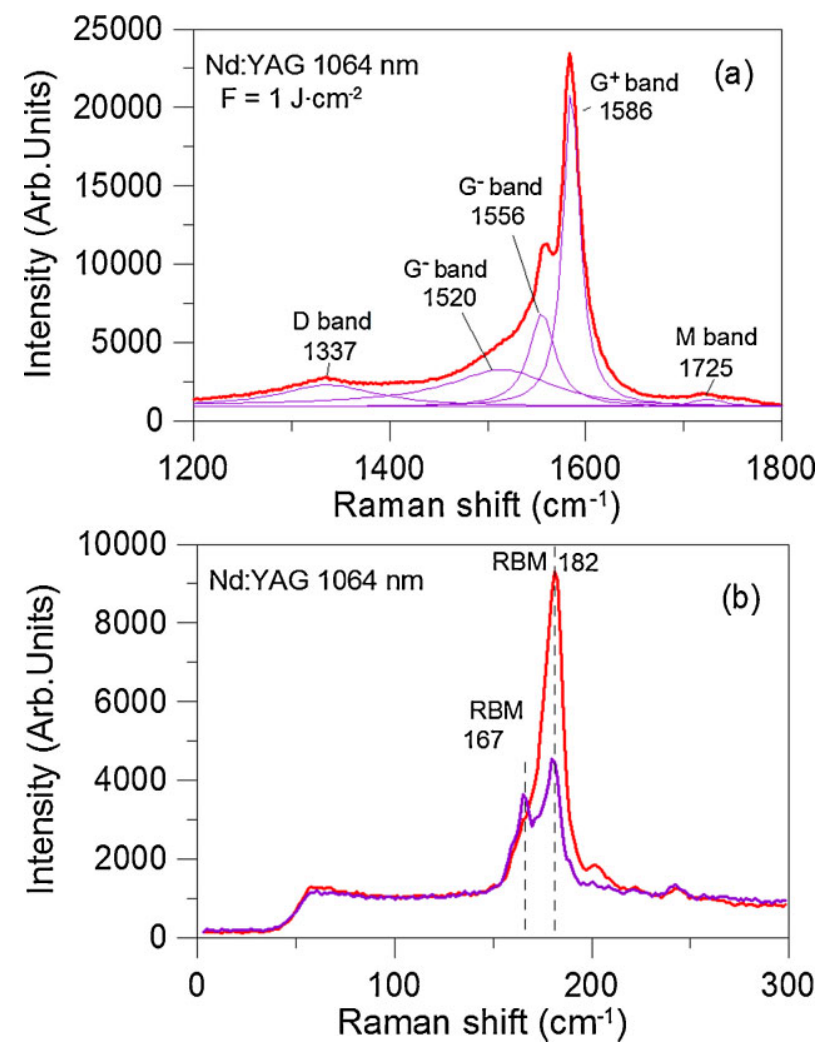

Figure 5 Raman shifts for PLV (configuration 2) with Nd:YAG $1064 \mathrm{~nm}$ at $F=1 \mathrm{~J} \cdot \mathrm{cm}^{-2}$; (a) deconvoluted $\mathrm{G}$ bands, (b) RBMs.

profile is narrow and Lorentzian, while in the case of metallic nanotubes, its profile is broader, asymmetric, and is described by a Breit-Wigner-Fano (BWF) line shape [25]. Metallic $\mathrm{G}^{-}$peaks are also shifted toward lower frequencies with respect to semi-conducting. Other line profiles are Lorentzian in both cases. The results of deconvolution presented in Fig. 4b show that the CNTs produced in the experiment are of mixed nature.

The ratio of intensities of semi-conducting (Lorentzian) to metallic (BWF) component is $I_{\mathrm{G}^{-}}^{\mathrm{L}} / I_{\mathrm{G}^{-}}^{\mathrm{BMF}}=0.21$. This value is only indicative. For quantitative analysis, the knowledge of the Raman excitation cross sections and radiative transition probabilities are necessary. With the use of well-known frequency vs. 1/diameter diagram by Jorio and colleagues [26], it has been found that the position of the peaks corresponds to the SWCNTs diameters of 1.16 and $1.1 \mathrm{~nm}$ for semi-conducting and metallic nanotubes, respectively.

Double RBM peak at frequencies of 183 and $204 \mathrm{~cm}^{-1}$ (Fig. 4c) indicates a narrow distribution of single-wall nanotubes with a dominant population of about 1.16 and $1.3 \mathrm{~nm}$ diameter according to the formula $\omega=227.0 / d+B$ $[27,28]$, where $\omega$ denotes the RBM peak position, $d$ the carbon nanotube diameter in nanometers, and $B$ is upshift from the pristine value due to van der Waals' interactions between bundled nanotubes (for negligible environmental effect $B \approx 0$ ). The value of $B \approx 9 \mathrm{~cm}^{-1}$ was taken from Fig. 3 of Ref. [27]. Simple relation $\omega=227.0 / d \cdot \sqrt{1+C \cdot d^{2}}$ with $C \approx 0.058[27,28]$ gives very similar results. The SWCNTs diameters determined from the frequency of RBM peaks are more reliable than those determined from the position of $\mathrm{G}^{-}$peaks; we will further rely on this approach.

Since it has been found $[26,29]$ that in the case of laser excitation energy $2.41 \mathrm{eV}$, only semi-conducting SWCNTs have resonant RBM frequencies between 160 and $220 \mathrm{~cm}^{-1}$, we may assume that both observed RBM peaks come from the semi-conducting SWCNTs.

Similar results were obtained in the double tube system (configuration 2). The Raman spectra of CNTs obtained at fluence $F=1 \mathrm{~J} \cdot \mathrm{cm}^{-2}$ are shown in Fig. 5 . The ratio of intensities of Lorentzian to BMF component is $I_{\mathrm{G}^{-}}^{\mathrm{L}} / I_{\mathrm{G}^{-}}^{\mathrm{BMF}}=0.3$. Again the dominant population has a diameter of $1.3 \mathrm{~nm}$ although in some cases another population with a diameter of $1.44 \mathrm{~nm}$ appears. At fluence $F=3 \mathrm{~J} \cdot \mathrm{cm}^{-2}$ (Fig. 6), the ratio of intensities of Lorentzian to $\mathrm{BMF}$ component is considerably lower, i.e., $I_{\mathrm{G}^{-}}^{\mathrm{L}} / I_{\mathrm{G}^{-}}^{\mathrm{BMF}}=0.13$, which indicates increased amount of metallic nanotubes. The most abundant population has diameter of $1.32 \mathrm{~nm}$ but diameter range is much broaderfrom 0.96 to $1.65 \mathrm{~nm}$ (Fig. 6b).

Conversely, at fluence $F=6 \mathrm{~J} \cdot \mathrm{cm}^{-2}$ (Fig. 7), $I_{\mathrm{G}^{-}}^{\mathrm{L}} / I_{\mathrm{G}^{-}}^{\mathrm{BMF}}=0.71$ which means that SWCNTs are
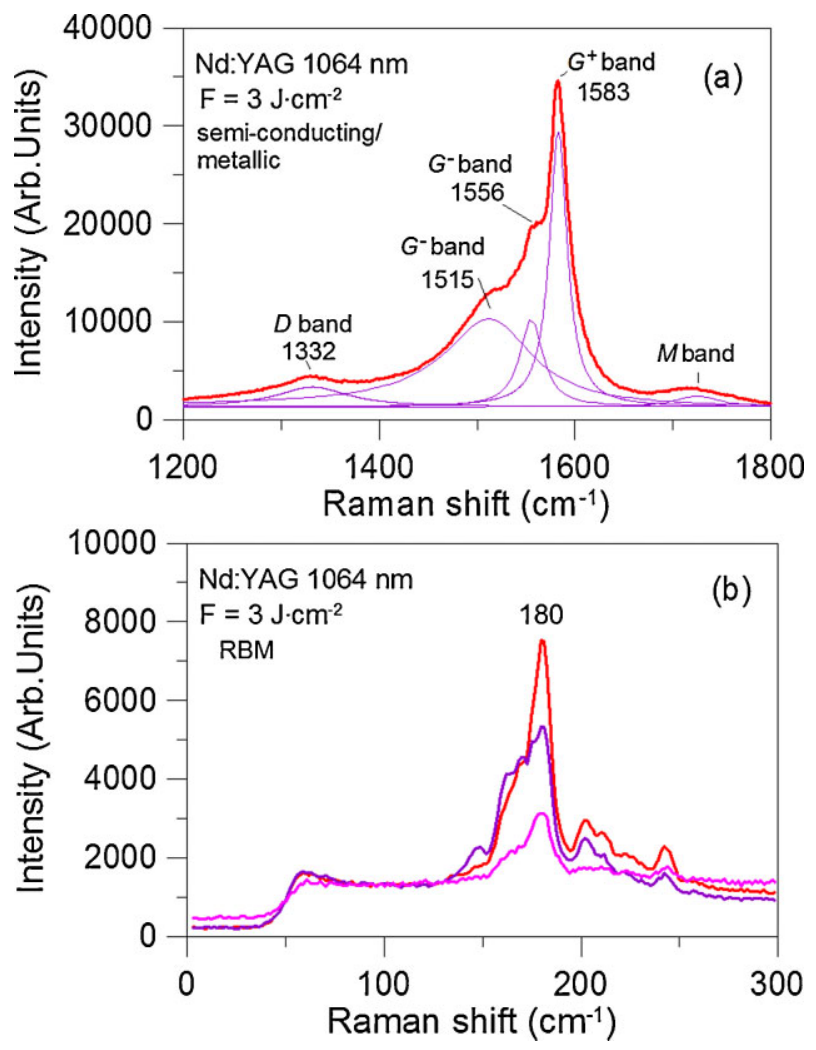

Figure 6 Raman shifts for PLV (configuration 2) with Nd:YAG $1064 \mathrm{~nm}$ at $F=3 \mathrm{~J} \cdot \mathrm{cm}^{-2}$; (a) deconvoluted $\mathrm{G}$ bands, (b) RBMs. 

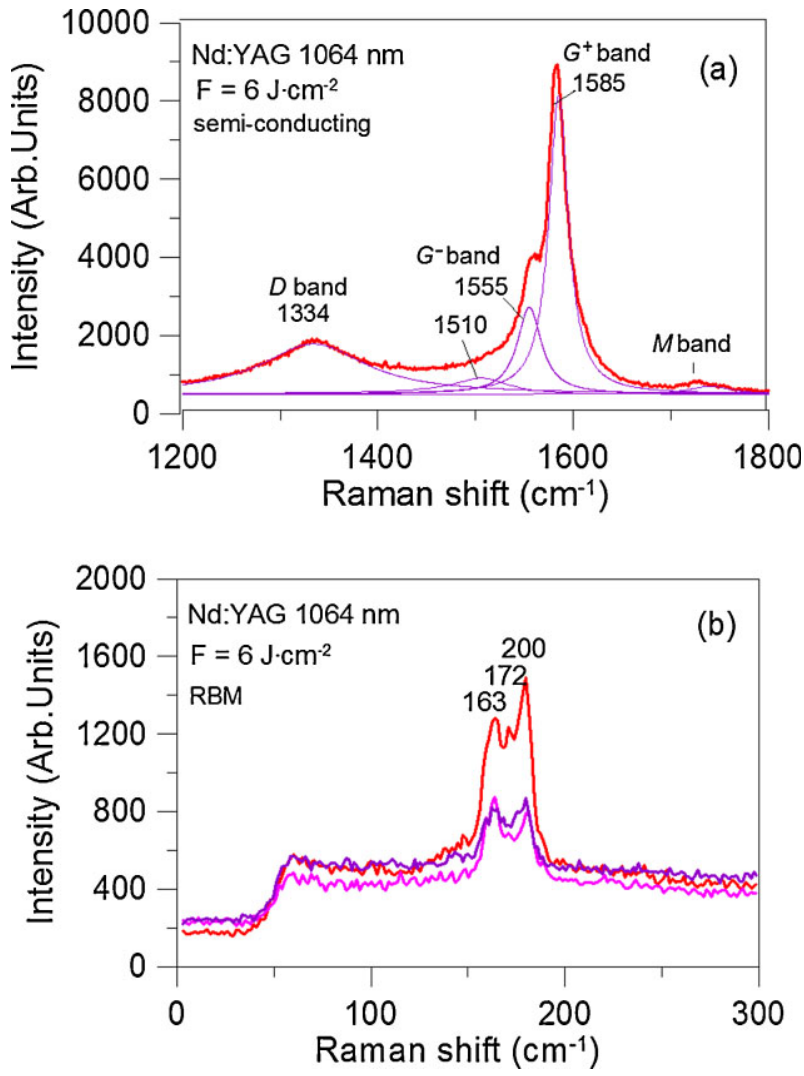

Figure 7 Raman shifts for PLV (configuration 2) with Nd:YAG $1064 \mathrm{~nm}$ at $F=6 \mathrm{~J} \cdot \mathrm{cm}^{-2}$; (a) deconvoluted $\mathrm{G}$ bands, (b) RBMs.

predominantly semi-conducting. In this case, the synthesis yield of nanotubes was significantly lower as was concluded from STEM images. The population becomes more diverse with increasing fluence; in the case of $F=6 \mathrm{~J} \cdot \mathrm{cm}^{-2}$, the most abundant nanotube diameters are 1.18, 1.39, and $1.48 \mathrm{~nm}$ (Fig. 7b).

The effect of laser influence on the production yield of CNTs has been studied in many papers. A comprehensive compendium of results obtained with the first and second harmonic (or combination of both) of Nd:YAG laser can be found in Ref. [30]. The results show that appropriate conditions for SWCNTs synthesis are typically found at fluence $1-3 \mathrm{~J} \cdot \mathrm{cm}^{-2}$. In the case of an Nd:YAP laser working at a wavelength of $1079 \mathrm{~nm}$, the conditions that led to the highest yield of SWCNTs were found at $F=1.5$ $\mathrm{J} \cdot \mathrm{cm}^{-2}$ while at $F=3 \mathrm{~J} \cdot \mathrm{cm}^{-2}$, about $40 \%$ decrease of production rate was observed [31]. However, some authors [32] report considerably higher optimum fluences, i.e., $F=8.5 \mathrm{~J} \cdot \mathrm{cm}^{-2}$ in the case of a single pulse $1064 \mathrm{~nm}$ wavelength and $F=17.5 \mathrm{~J} \cdot \mathrm{cm}^{-2}$ in the case of a single pulse $532 \mathrm{~nm}$ wavelength. Our results show that for a double pulse at a wavelength of $1064 \mathrm{~nm}$, the most favorable conditions for a high production yield of SWCNTs are at fluences $1 \leq F \leq 3$, where $F$ is the fluence of each of two pulses. The estimates are based on STEM images. Since such estimations are quite subjective, the absolute abundances of SWCNTs were not determined.

3.2 Laser wavelength of $355 \mathrm{~nm}$ In the case of CNTs obtained with a laser wavelength of $355 \mathrm{~nm}$ at fluence $F=1 \mathrm{~J} \cdot \mathrm{cm}^{-2}$ (Fig. 8), the position and shape of very broad BMF component at $\omega_{\mathrm{G}}^{-} \sim 1530 \mathrm{~cm}^{-1}$ indicates the metallic nature of nanotubes. The RBM peak consists of several very small peaks in the range of $166-222 \mathrm{~cm}^{-1}$. All RBM peaks
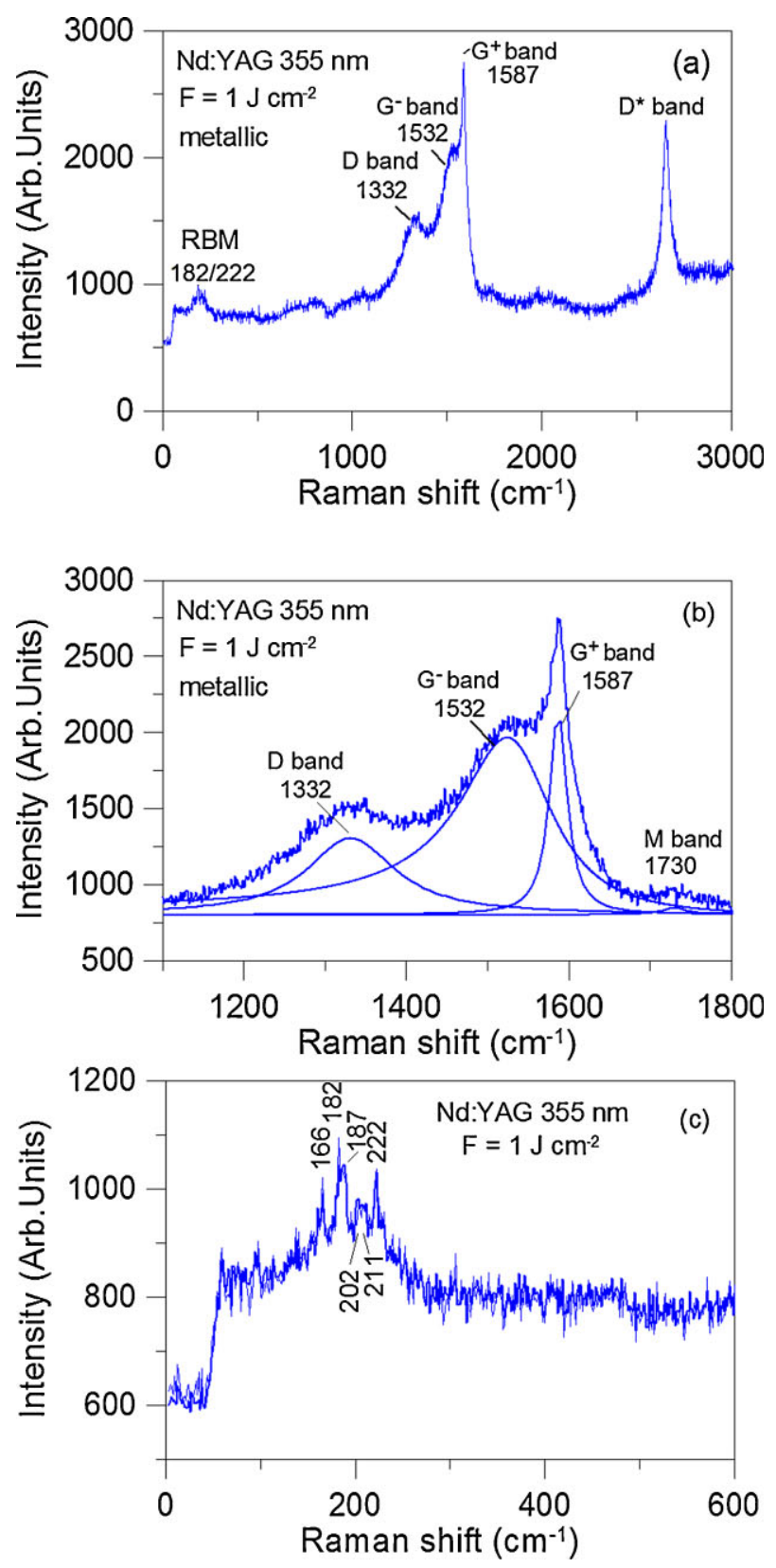

Figure 8 Raman shifts for PLV (configuration 2) with Nd:YAG at $355 \mathrm{~nm}$ at $F=1 \mathrm{~J} \cdot \mathrm{cm}^{-2}$; (a) full spectrum, (b) deconvoluted $\mathrm{G}$ bands; thick line-experiment, thin lines-separated spectrum, (c) RBMs. 
(except peak at $222 \mathrm{~cm}^{-1}$ ) probably belong to residual semiconducting SWCNTs, because metallic RBM peaks are not excited by $514 \mathrm{~nm}$ wavelength below $220 \mathrm{~cm}^{-1}$. The intensity of the D-band (disorder band) in relation to the G-band increases, which means much higher level of structure disorder. This could mean either defects in the nanotube lattice and/or enlarged level of amorphous carbon in the sample. Indeed, the SEM/STEM images of the collected soot show much lower output of carbon nanotubes in comparison to the results obtained at similar fluence with a laser wavelength of $1064 \mathrm{~nm}$.

Satisfying results were obtained at fluence $F=3 \mathrm{~J} \cdot \mathrm{cm}^{-2}$ (Fig. 9) which means that in the case of UV radiation, much higher fluence is necessary for reasonable SWCNTs yield. The results are similar to those presented in Fig. 6 in the case of $1064 \mathrm{~nm}$ wavelength, although the ratio of intensities of semi-conducting to metallic component is somewhat lower, $I_{\mathrm{G}^{-}}^{\mathrm{L}} / I_{\mathrm{G}^{-}}^{\mathrm{BMF}}=0.64$. A double RBM peak at frequency of 183 and $202 \mathrm{~cm}^{-1}$ points to a narrow distribution of single-wall nanotubes with the dominant population of 1.17 and $1.3 \mathrm{~nm}$ diameter. The production yield was similar to that observed in the case of $1064 \mathrm{~nm}$ radiation.

Indeed, in the case of a $\mathrm{KrF}$ laser with a wavelength of $248 \mathrm{~nm}$ [9], the fluence at which high quality SWCNTs were produced was $5.25 \mathrm{~J} \cdot \mathrm{cm}^{-2}$. Similarly, in the case of excimer $\mathrm{XeCl}$ laser with a wavelength of $308 \mathrm{~nm}$ [33], fluence of $2.25 \mathrm{~J} \cdot \mathrm{cm}^{-2}$ was needed.

The Raman spectra obtained from materials synthesized at $F=6 \mathrm{~J} \cdot \mathrm{cm}^{-2}$ are shown in Fig. 10. Since excitation with $514 \mathrm{~nm}$ does not reveal any RBM peaks, the Raman spectra were repeated using $633 \mathrm{~nm}$ excitation source showing RBM peak at $188 \mathrm{~cm}^{-1}$ corresponding to the SWCNTs diameter of $1.26 \mathrm{~nm}$. The $\mathrm{G}$ band consists of one peak only, which shows a BWF line shape, although the fit to the experimental data was not the best. The absence of broad $\mathrm{G}^{-}$ component was already observed [34] in the case of metallic armchair structures. More importantly, the production rate of SWCNTs is substantially reduced. The STEM and HRTEM images demonstrate that nanotubes are embedded in a large amount of amorphous carbon. The results show that the appropriate fluence range for the production of SWCNTs is much narrower and the synthesis products depend much more on the laser fluence in the case of $355 \mathrm{~nm}$ than in the case of $1064 \mathrm{~nm}$.

The upper limit of fluence for the $355 \mathrm{~nm}$ wavelength can be restricted by the phase explosion threshold. This threshold corresponds to a sudden transition from surface vaporization (thermal ablation) to explosive boiling (also known as phase explosion). Rapid laser heating causes the heated liquid-vapor system to leave the saturation line and enter the metastable region. When a liquid reaches its homogeneous nucleation temperature $T_{\mathrm{hn}} \geq 0.9 T_{\mathrm{cr}}$ (where $T_{\text {cr }}$ denotes critical temperature), rapid transition from a superheated liquid to a mixture of vapor and liquid occurs. Since the volume of the gas phase greatly exceeds that of the liquid phase, sudden boiling resembles an explosion [35] and results in the ejection of a mixture of vapor and liquid droplets. It has been found [36] that the phase transition threshold strongly depends on the laser wavelength and decreases from $F=55 \mathrm{~J} \cdot \mathrm{cm}^{-2}$ for $1064 \mathrm{~nm}$ to $F=7.5 \mathrm{~J} \cdot \mathrm{cm}^{-2}$ for $355 \mathrm{~nm}$. These data concern a Goodfellow rigid foil target and can be somewhat different for various carbon targets. Therefore, it is plausible that in the case of $355 \mathrm{~nm}$ at laser fluence $F=6 \mathrm{~J} \cdot \mathrm{cm}^{-2}$, the synthesis conditions were already affected by the phase explosion.

The higher threshold of fluence for production of CNTs in the case of $355 \mathrm{~nm}$ wavelength in comparison to
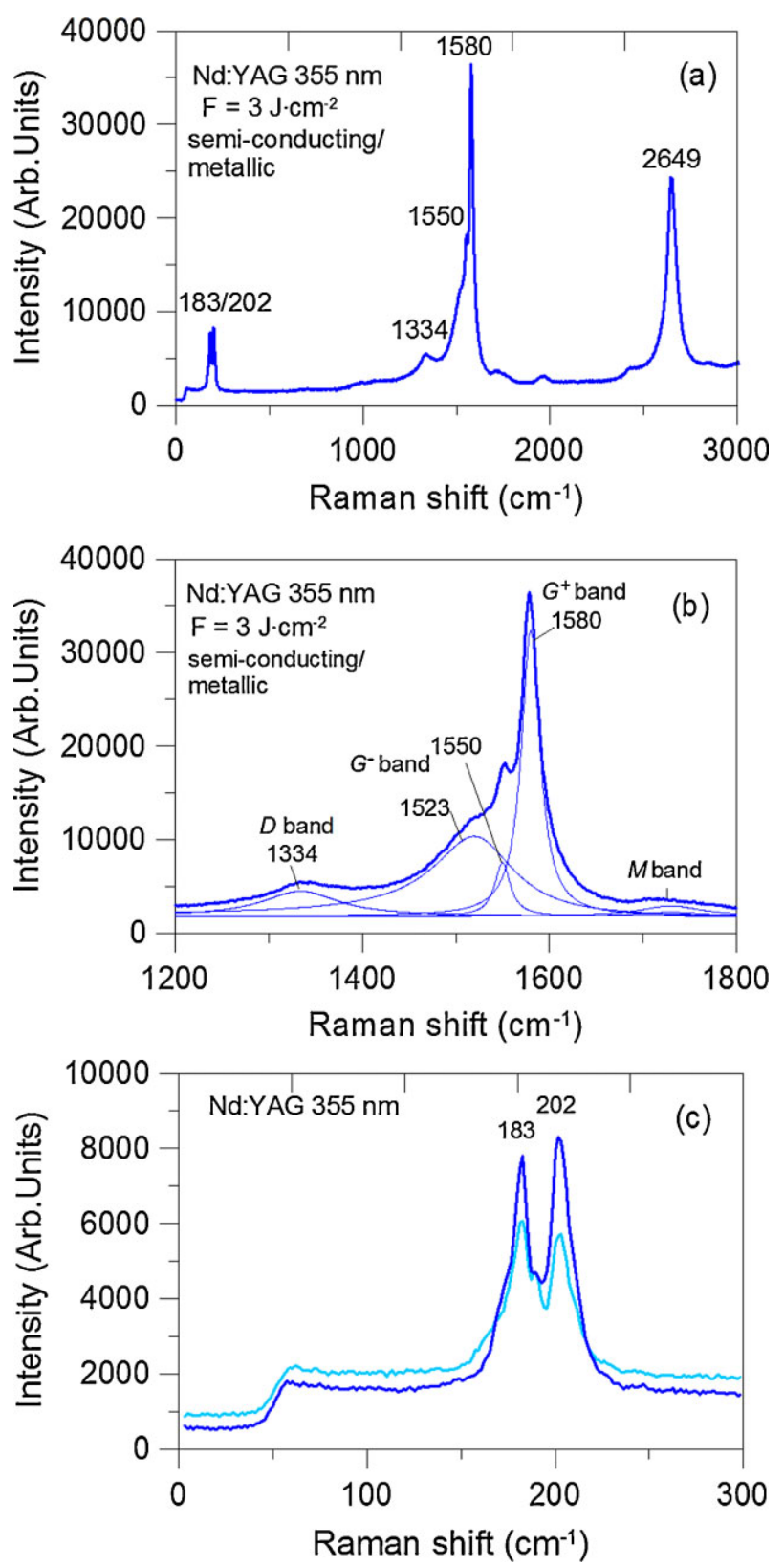

Figure 9 Raman shifts for PLV (configuration 2) with Nd:YAG at $355 \mathrm{~nm}$ at $F=3 \mathrm{~J} \cdot \mathrm{cm}^{-2}$; (a) full spectrum, (b) deconvoluted $\mathrm{G}$ bands; thick line - experiment, thin lines—separated spectrum, (c) RBMs. 

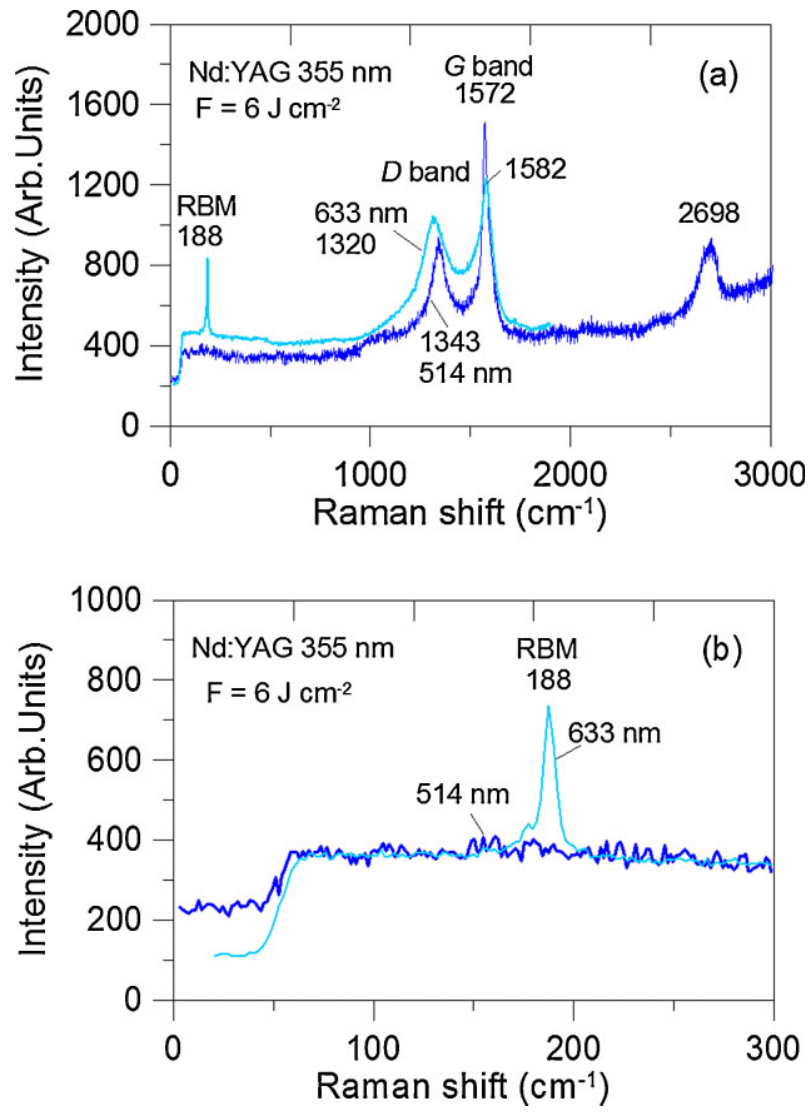

Figure 10 Raman shifts for PLV (configuration 2) with Nd:YAG at $355 \mathrm{~nm}$ at $F=6 \mathrm{~J} \cdot \mathrm{cm}^{-2}$; (a) full spectrum, (b) RBMs.

$1064 \mathrm{~nm}$ is more difficult to explain given the large number of factors controlling the efficiency of nanotube synthesis. Since the main effect of laser wavelength on laser-induced carbon plasma [36, 37] is the lower temperature in the case of lower wavelength, we can assume that in the case of $355 \mathrm{~nm}$ fluence of $1 \mathrm{~J} \cdot \mathrm{cm}^{-2}$ is probably too low to create appropriate conditions for nanotube synthesis.

4 Conclusions A double-pulse laser system for high yield single-wall CNTs synthesis was used. The results show that the growth conditions change considerably with the laser fluence. The results show that the useful range of the UV laser radiation fluence is narrower and the properties of synthesized nanotubes depend much more on the laser fluence than in the case of infrared laser radiation. For $355 \mathrm{~nm}$ laser wavelength, good quality SWCNTs were obtained only at fluence $F=3 \mathrm{~J} \cdot \mathrm{cm}^{-2}$ while for $1064 \mathrm{~nm}$ good results were obtained in the fluence range of $1 \leq F \leq 6 \mathrm{~J} \cdot \mathrm{cm}^{-2}$, although the production yield decreased for $F>3 \mathrm{~J} \cdot \mathrm{cm}^{-2}$. In the case of $1064 \mathrm{~nm}$ the population distribution for SWCNTs was the narrowest at fluence $F=1 \mathrm{~J} \cdot \mathrm{cm}^{-2}$ and then the most abundant population of CNTs had a diameter of $1.3 \mathrm{~nm}$. The population distribution becomes broader with increasing fluence, encompassing a larger range of SWCNTs diameters.
Acknowledgements This work was supported by the Polish National Science Centre (Research Project 2014/13/B/ST8/ 04290). The authors (L.S., A.M., M.M.) are also thankful for support of the National Center for Research and Development: PBS1/A5/15/2012.

\section{References}

[1] T. Yoshitake, T. Nishiyama, H. Aoki, K. Suizu, K. Takahashi, and K. Nagayama, Diam. Relat. Mater. 8, 463 (1999).

[2] J. Hunter, J. Fye, and M. F. Jarrold, Science 260, 784 (1993).

[3] A. Thess, R. Lee, P. Nikolaev, H. Dai, P. Petit, J. Robert, Ch. $\mathrm{Xu}$, Y. H. Lee, S. Gon, Kim, A. G. Rinzler, D. T. Colbert, G. E. Scuseria, D. Tomanek, J. E. Fischer, and R. E. Smalley, Science 273, 483-487 (1996).

[4] A. C. Dillon, P. A. Parilla, K. M. Jones, G. Riker, and M. J. Heben, Mater. Res. Soc. Symp. Proc. 526, 403-408 (1998).

[5] H. Kataura, Y. Kumazawa, Y. Maniwa, Y. Ohtsuka, R. Sen, S. Suzuki, and Y. Achiba, Carbon 38, 1691-1697 (2000).

[6] A. A. Puretzky, H. Schittenhelm, X. Fan, M. J. Lance, F. L. Allard Jr., and D. B. Geohegan, Phys. Rev. B 65, 245425 (2002).

[7] P. Nikolaev, W. Holmes, E. Sosa, P. Boul, S. Arepalli, and L. Yowell, J. Nanosci. Nanotechnol. 10, 3780 (2010).

[8] C. D. Scott, S. Arepalli, P. Nikolaev, and R. E. Smalley, Appl. Phys. A 72, 573 (2001).

[9] N. Braidy, M. A. El Khakani, and G. A. Botton, Chem. Phys. Lett. 354, 88 (2002).

[10] F. Kokai, K. Takahashi, M. Yudasaka, and S. Iijima, J. Phys. Chem. B 104, 6777 (2000).

[11] G. Compagnini, M. Sinatra, P. Russo, G. C. Messina, O. Puglisi, and S. Scalese, Carbon 50, 2347 (2012).

[12] A. A. Puretzky, D. J. Styers-Barnett, C. M. Rouleau, H. Hu, B. Zhao, I. N. Ivanov, and D. B. Geohegan, Appl. Phys. A 89, 121 (2007).

[13] S. Z. Mortazavi, P. Parvin, and A. Reyhani, Laser Phys. Lett. 9, 547 (2012).

[14] T. T. Koh, Y. M. Foong, and D. H. C. Chua, Appl. Phys. Lett. 97, 114102 (2010).

[15] M. Qian, Y. S. Zhou, Y. Gao, J. B. Park, T. Feng, S. M. Huang, Z. Sun, L. Jiang, and Y. F. Lu, Appl. Phys. Lett. 98, 173108 (2011).

[16] M. Qian, Y. S. Zhou, Y. Gao, T. Feng, Z. Sun, L. Jiang, and Y. F. Lu, Appl. Surf. Sci. 258, 9092 (2012).

[17] D. B. Geohegan, A. A. Puretzky, M. Yoon, G. Eres, C. Rouleau, K. Xiao, J. Jackson, J. Readle, M. Regmi, N. Thonnard, G. Duscher, M. Chisholm, and K. More, in: Lasers in Materials Science, edited by M. Castillejo, P. M. Ossi, and L. Zhigilei, Springer Series in Materials Science, Vol. 191 (Springer International Publishing, Switzerland, 2014), chap. 7.

[18] J. Leszczynski and M. K. Shukla (eds.), Practical Aspects of Computational Chemistry II (Springer Science + Business Media, Dordrecht, 2012).

[19] Y. F. Hsiou, Y. J. Yang, L. Stobinski, W. Kuo, and C. D. Chen, Appl. Phys. Lett. 84, 984-986 (2004).

[20] W. K. Maser, E. Muñoz, A. M. Benito, M. T. Martinez, G. F. de la Fuente, Y. Maniette, E. Anglaret, and J.-L. Sauvajol, Chem. Phys. Lett. 292, 587-593 (1998).

[21] E. Muñoz, W. K. Maser, A. M. Benito, M. T. Martinez, G. F. de la Fuente, A. Righi, J. L. Sauvajol, E. Anglaret, and Y. Maniette, Appl. Phys. A 70, 145-151 (2000). 
[22] A. C. Dillon, P. A. Parilla, J. L. Alleman, J. D. Perkins, and M. J. Heben, Chem. Phys. Lett. 316, 13-18 (2000).

[23] E. G. Gamaly, A. V. Rode, W. K. Maser, E. Muñoz, A. M. Benito, M. T. Martinez, and G. F. de la Fuente, Appl. Phys. A 70, 161-168 (2000).

[24] F. Kokai, K. Takahashi, K. Shimizu, M. Yudasaka, and S. Iijima, Appl. Phys. A 69, S223-S227 (1999).

[25] M. S. Dresselhaus, G. Dresselhaus, R. Saito, and A. Jorio, Phys. Rep. 409, 47 (2005).

[26] A. Jorio, A. G. Souza Filho, G. Dresselhaus, M. S. Dresselhaus, A. Swan, M. Ünlü, B. Goldberg, M. A. Pimenta, J. Hafner, C. M. Lieber, and R. Saito, Phys. Rev. B 65, 155412 (2002).

[27] P. T. Araujo, I. O. Maciel, P. B. C. Pesce, M. A. Pimenta, S. K. Doorn, H. Qian, A. Hartschuh, M. Steiner, L. Grigorian, K. Hata, and A. Jorio, Phys. Rev. B 77, 241403(R) (2008).

[28] R. Saito, M. Hofmann, G. Dresselhaus, A. Jorio, and M. S. Dresselhaus, Adv. Phys. 60, 413-550 (2011).

[29] R. Krupke, F. Hennrich, H. v. Lohneysen, and M. M. Kappes, Science 301, 344-347 (2003).
[30] A. P. Moravsky, E. M. Wexler, and R. O. Loutfy, in: Carbon Nanotubes: Science and Application, edited by M. Meyyappan (CRC Press, Boca Raton, FL, 2004).

[31] A. A. Gorbunov, R. Friedlein, O. Jost, M. S. Golden, J. Fink, and W. Pompe, Appl. Phys. A 69 (Suppl.), S593-S596 (1999).

[32] N. Braidy, M. A. El Khakani, and G. A. Botton, Carbon 40, 2835-2842 (2002).

[33] M. Kusaba and Y. Tsunawaki, Thin Solid Films 506-507, 255 (2006).

[34] E. H. Haroz, J. G. Duque, W. D. Rice, C. G. Densmore, J. Kono, and S. K. Doorn, Phys. Rev. B 84, 121403(R) (2011).

[35] P. G. Debenedetti, Metastable Liquids: Concepts and Principles (Princeton University Press, Princeton, 1996).

[36] J. Hoffman, J. Chrzanowska, S. Kucharski, T. Moscicki, I. N. Mihailescu, C. Ristoscu, and Z. Szymanski, Appl. Phys. A 117, 395 (2014).

[37] T. Moscicki, J. Hoffman, and Z. Szymanski, J. Appl. Phys. 114, 083306 (2013). 